\title{
Aspectos clínicos, ultra-sonográficos e venográficos da trom- boflebite jugular experimental em equinos ${ }^{1}$
}

\author{
Carlos Alberto Hussni ${ }^{2 *}$, Roberta Galvano Barbosa ${ }^{3}$, Alexandre Correa Borghesan ${ }^{3}$, \\ Hamilton Almeida Rollo ${ }^{4}$, Ana Liz Garcia Alves ${ }^{2}$, Marcos Jun Watanabe ${ }^{2}$, Vania \\ Maria de Vasconcelos Machado ${ }^{5}$ e Nereide Freire Cerqueira ${ }^{3}$
}

\begin{abstract}
Hussni C.A., Barbosa R.G., Borghesan A.C., Rollo H.A., Alves A.L.G., Watanabe M.J., Machado V.M.V. \& Cerqueira N.F. 2012. [Equine experimental thrombophlebitis: Clinical, ultrasonographic and venographic evaluation.] Aspectos clínicos, ultra-sonográficos e venográficos da tromboflebite jugular experimental em equinos. Pesquisa Veterinária Brasileira 32(7):595-600. Departamento de Cirurgia e Anestesiologia Veterinária, Faculdade de Medicina Veterinária e Zootecnia, Universidade Estadual Paulista, Rubião Jr s/n, Botucatu, SP 18618970, Brazil. E-mail: cahussni@fmvz.unesp.br

Jugular thrombophlebitis is a common complication of disease processes associated with repeated venipuncture, injection of irritant solutions, and the use of indwelling catheters, especially with bacterial contamination. Bilateral thrombophlebitis may result in edema of the soft tissues of the head, reduction of athletic performance and even death of the animal. This disease, although common in horses, is not much known regarding its evolution and treatment. The aim of this study was to evaluate the clinical and structural changes of experimentally induced jugular thrombophlebitis in horses, through clinical examination, ultrasound and venography of the thrombus and the vessel, verifying the possibility of thrombus recanalization and compensatory produced blood flow. The jugular thrombophlebitis was induced unilaterally into 5 horses, monitored by clinical (general, regional and local) and ultrassonographycs exams. Venographs were made at pre-induction, induction and every 6 days after induction of thrombophlebitis, in order to observe recanalization of the occlusive thrombus and presence of blood vessels in the drainage allowance. Occurrence of moderate edema was observed in the parotid, masseter and supra orbital regions, and mild edema in the submandibular region. The jugular engorgement of the cranial region of induction persisted throughout the period of evaluation. The caudal portion to the thrombophlebitis showed engorgement with compression on the vein at the thorax entrance since the first day after induction. The ultrasound examinations showed total occlusive thrombus formation of 3 animals, partial recirculating flow in the jugular vein in 2 animals, and collateral blood vessels from the cranial obstruction to the caudal portion. The venography revealed normal linear blood flow in the preoperative and occlusive thrombus with contrast directed filling of the vessels to the compensatory portion caudal to the vein occlusion or cranial to the thrombus in the postoperative moments. After vein resection of the segment containing the thrombus, the cephalic edema was less intense than after the induction of the thrombophlebtits. The ultrassonography and venography post resection showed vascularity increase in this region. It was concluded that there is recanalization with endothelialization and vascular compensation made by pre-existing vessels necessary for drainage.
\end{abstract}

INDEX TERMS: Horses, jugular, thrombophlebitis, recanalization.

\footnotetext{
${ }^{1}$ Recebido em 25 de abril de 2011.

Aceito para publicação em 7 de novembro de 2012.

${ }^{2}$ Departamento de Cirurgia e Anestesiologia Veterinária, Faculdade de Medicina Veterinária e Zootecnia (FMVZ), Universidade Estadual Paulista (Unesp), Rubião Jr s/n, Botucatu, SP 18618970, Brasil. *Autor para correspondência: cahussni@fmvz.unesp.br
}

\footnotetext{
${ }^{3}$ Programa de Pós-Graduação em Medicina Veterinária, FMVZ-Unesp, Botucatu, SP.

${ }^{4}$ Departamento de Cirurgia e Ortopedia, Faculdade de Medicina, Unesp, Rubião Jr s/n, Botucatu, SP 18618970.

${ }^{5}$ Departamento de Reprodução Animal e Radiologia Veterinária, FMVZ-Unesp, Botucatu, SP.
} 
RESUMO.- A tromboflebite jugular ocorre frequentemente em equinos, decorrendo geralmente de processos mórbidos associados à iatrogenia, podendo levar a perda de função, edema cefálico, diminuição do desempenho atlético e ainda causar o óbito. Esta enfermidade nos equinos apesar de frequente é pouco conhecida quanto à sua evolução e tratamentos. 0 objetivo deste trabalho foi avaliar a evolução da tromboflebite jugular experimental em equinos, quanto às alterações clínicas e estruturais envolvidas na enfermidade, observando-se os aspectos clínicos, ultra-sonográficos e venográficos no contexto do trombo e do vaso, quanto à possibilidade de recanalização do trombo produzido e da vascularização compensatória. A tromboflebite da veia jugular foi induzida, unilateralmente, em 05 equinos nos quais previamente à indução da tromboflebite e diariamente após foram observadas manifestações clínicas e realizados exames ultra-sonográficos. Venografias foram feitas nos momentos pré-indução, na indução e a cada seis dias após a indução da tromboflebite, verificando-se a recanalização do trombo oclusivo e a presença de vasos na drenagem sanguínea compensatória. Observou-se a ocorrência de edema moderado das regiões parotídea, massetérica e supra-orbitária e discreto edema submandibular que reduziram até o $6^{\circ}$ dia, permanecendo apenas discreto aumento parotídeo. 0 ingurgitamento da jugular cranial a região da indução permaneceu durante todo o período de avaliação. A porção caudal à tromboflebite mostrou ingurgitamento frente ao garrote na entrada do tórax desde o primeiro dia após a indução. Os exames ultra-sonográficos mostraram formação de trombo oclusivo total durante todo o período de observação em 3 animais e o restabelecimento parcial do fluxo na jugular em 2 animais e a presença de vasos colaterais conduzindo o sangue da porção cranial para a porção caudal à obstrução. As venografias revelaram fluxo sanguíneo "linear" normal no momento pré-operatório, constatando nos momentos pós-operatórios a presença oclusiva do trombo, com o contraste preenchendo os vasos tributários compensatórios direcionados à porção caudal à oclusão da veia ou ainda estagnado cranialmente ao trombo. Conclui-se que a trombose oclusiva na tromboflebite jugular experimental e unilateral sofre recanalização e compensação vascular por vasos tributários de drenagem, com redução gradativa dos sinais decorrentes da estase sanguínea de retorno, especificamente as regiões cefálicas com edema. Estudos envolvendo a tromboflebite jugular nos equinos devem evoluir nos aspectos experimental e clínico.

TERMOS DE INDEXAÇÃO: Equinos, jugular, tromboflebite, recanalização.

\section{INTRODUÇÃO}

A ocorrência de trombose da veia jugular na espécie equina constitui a formação e desenvolvimento de trombo que pode ser concebido como coágulo sanguíneo formado na luz venosa (Montenegro \& Franco 1999), geralmente associada a processos iatrogênicos resultantes da complicação de venopunções repetitivas, aplicação de cateteres, medicação intravenosa com fluxo rápido por longo período, o uso de substâncias e medicamentos agressivos ao endoté- lio vascular e a deposição perivascular de medicamentos que por si só poderiam levar a tromboflebite (Dornbusch et al. 2000, Wiemer et al. 2005).

A tromboflebite jugular em equinos pode resultar em oclusão completa da veia. Nestes casos o fluxo sanguíneo poderá ser restaurado pela circulação colateral ou pela recanalização. Com a ausência da veia jugular interna, o desenvolvimento de circulação colateral é dificultado. Quando a capacidade da nova circulação permanece insuficiente, o desempenho atlético do cavalo diminui (Rijkenhuizen \& Van Swieten 1998, Wiemer et al 2005). Nos casos agudos e bilaterais há diminuição da drenagem venosa da região cefálica com conseqüente edema de cabeça, edema de faringe, de laringe resultando na obstrução das vias aéreas e edema cerebral, podendo levar o paciente à morte (Morris 1989, Hay 1998, Rijkenhuizen \& Van Swieten 1998, Dornbusch et al. 2000, Hussni et al. 2009).

Apesar de frequente nesta espécie a tromboflebite jugular é pouco conhecida quanto a sua evolução. Este trabalho teve por objetivo estudar a evolução da tromboflebite jugular unilateral experimentalmente induzida em equinos em seus aspectos clínicos, ultra-sonográficos e venográficos.

\section{MATERIAL E METODOS}

Cinco animais de idade entre cinco e sete anos, mestiços com 350 quilos de peso médio, de ambos os sexos foram submetidos a indução experimental da tromboflebite jugular direita com aplicação intraluminal de glicose $50 \%$ após dissecção e estase segmentar do vaso com ponto estenosante na porção média da região cervical (Hussni et al 2009). A indução da tromboflebite consistiu em período de três dias com manutenção por este período de um ponto de sutura estenosante destinado a manter o trombo na luz vascular. Diariamente foram realizados exames físicos gerais (temperatura corpórea, freqüências cardíaca e respiratória, coloração das mucosas e tempo de reperfusão capilar), regionais quanto a alterações cefálicas e cervicais e locais sobre a veia jugular direita, bem como os exames ultra-sonográficos. Estes tiveram início imediatamente antes da indução da tromboflebite, período de indução (72 horas) até o 18o dia após induzida a tromboflebite. Exames venográficos foram realizados antes da indução, no termino do período de indução (72 horas) e repetiu-se a cada 6 dias após induzida a tromboflebite (dias 6,12 e 18). Os exames prévios ao início dos procedimentos confirmaram a higidez dos animais dada especial atenção a veia jugular direita.

Os animais foram submetidos aos exames físicos diários com avaliação da temperatura retal, frequencias cardíaca e respiratória, auscultação torácica, coloração das mucosas e tempo de preenchimento capilar, observados aspectos cefálicos quanto à ocorrência de edema, aumento de volume e ingurgitamento de vasos principalmente nas regiões massetéricas, supra-orbitárias, submandibular e parotídeas. Aspectos clínicos locais foram considerados quanto a veia jugular com tromboflebite, na ocorrência de edema, aumento de volume, temperatura local, sensibilidade dolorosa ao toque e avaliação do fluxo sanguíneo com observação do ingurgitamento da veia com a compressão digital na transição cérvico-torácica.

Avaliações ultra-sonográficas da veia jugular direita foram realizadas diariamente antes e após a indução da tromboflebite, utilizando-se transdutor linear de 7,5 MHz modo B (Aloka SSD900, Japan), observados os aspectos de regularidade do fluxo sanguíneo presente, nas porções, cranial ao trombo, na região da cirurgia e caudal a estenose, bem como as características individuais 
dos diferentes segmentos, considerando-se os critérios presença de fluxo, presença, comprimento e características do trombo, e compressibilidade venosa frente à pressão exercida pelo transdutor do ultra-som e a presença de vasos colaterais além das partes do trombo (cabeça, corpo e cauda) (Hussni et al. 2009).

Venografias da jugular submetida à tromboflebite foram realizadas nos momentos pré-indução, na indução e repetidas no 6을 $12^{\circ}$ e $18^{\circ}$ dias. Com o animal em apoio quadrupedal foram procedidas com aparelho portátil (CTI 90 Plus - Electra, Rio de Janeiro), posicionado com projeção látero-lateral da esquerda para a direita. 0 contraste radiográfico por $20 \mathrm{ml}$ de diatrizoato de meglumina (Omnipaque-Reliev 60\%, Berlimed/Justesa, Rio de Janeiro) foi aplicado por meio de escalpe introduzido na veia jugular próximo à bifurcação cranial da veia jugular. Utilizando-se as venografias observou-se a presença de fluxo, com a oclusão luminal vascular pelo trombo foi feita contagem dos vasos, nos diferentes momentos, vasos estes que saiam da jugular a partir da sua origem na bifurcação das veias linguofacial com a maxilar até a porção caudal ao segmento com trombose.

Este trabalho está de acordo com os Princípios Éticos na Experimentação Animal, aprovado pela Câmara de Ética em Experimentação Animal da Faculdade de Medicina Veterinária e Zootecnia, Unesp, sob o número de protocolo 12/2007.

\section{RESULTADOS}

As frequencias cardíaca e respiratória, a temperatura corporal, o tempo de perfusão capilar e a coloração das mucosas mantiveram-se normais, em todos os animais, durante todo o período.

Houve aumento de volume das regiões massetérica, supra-orbitária, submandibular e parotídea observáveis imediatamente após a indução seguida de redução deste aumento de volume. Entre os dias 7 e 10 observou-se tendência de aumento de volume com as regiões massetérica e submandibular sutilmente aumentadas e que se mantiveram com edema até o 18ํ dia de observação.

As avaliações clínicas da região cervical, especificamente a veia jugular e estruturas adjacentes, mostraram aumento de volume na região da indução cirúrgica da tromboflebite, com discreta sensibilidade dolorosa e discreto aumento da temperatura local estimados à palpação que reduziram gradativamente até o 5 dia deste período, permanecendo leve reação cicatricial da pele que se organizou até o dia 13 e então não mais observável. Houve tendência de crescimento dos trombos até próximo à união das veias linguofacial e maxilar, ou acima, fazendo com que a jugular se mantivesse distendida, firme e não cedesse à compressão. Com isso a veia linguofacial permaneceu distendida. Apenas um animal, a partir do 13ำ dia mostrou diminuição da distensão da veia jugular na transição proximal/média cervical, que cedia a compressão a partir do dia 12. Apenas dois animais tiveram discreta distensão de vasos subcutâneos na região do sulco jugular. A realização de garrote na entrada do tórax mostrava presença fluxo das porções cranial para caudal da veia com trombo, com o ingurgitamento da veia jugular caudal à tromboflebite em todos os animais, mantendo também o ingurgitamento cranial ao trombo em um animal.

Os exames ultra-sonográficos da veia jugular direita revelaram fluxo laminar esperado em animais hígidos observado no momento pré-indução. Na indução e estabelecida a tromboflebite observou-se o fluxo de sangue com turbilhonamento cranial ao trombo também observado, com fluxo laminar caudal ao trombo. Na avaliação diária os trombos demonstraram tendência a continuar crescendo contra o sentido de fluxo sanguíneo da veia jugular. Concomitantemente ao crescimento do trombo observaram-se alterações do fluxo com a circunscrição da porção caudal do trombo pelo sangue com preenchimento com sangue entre o trombo e a parede vascular (Fig.1).

O comprimento máximo dos trombos variou entre 18 e $40 \mathrm{~cm}$ (média $29 \mathrm{~cm}$ ). Em três animais a trombose ascendeu alcançando as veias maxilar e linguofacial. 0 comprimento do trombo se fez com parte deste produzindo oclusão luminal total. No maior comprimento oclusivo total, foi mensurada oclusão de $22 \mathrm{~cm}$ em trombo de $40 \mathrm{~cm}$. Observou-se a passagem de sangue entre a parede vascular em casos com o trombo formado, com aumento de fluxo progressivo em outros casos observada esta evolução com o tempo de observação. A lise dos trombos era observável permitindo a passagem de sangue por pontos através do trombo ou entre a parede e este caracterizando a existência de canais, concebido como recanalização.
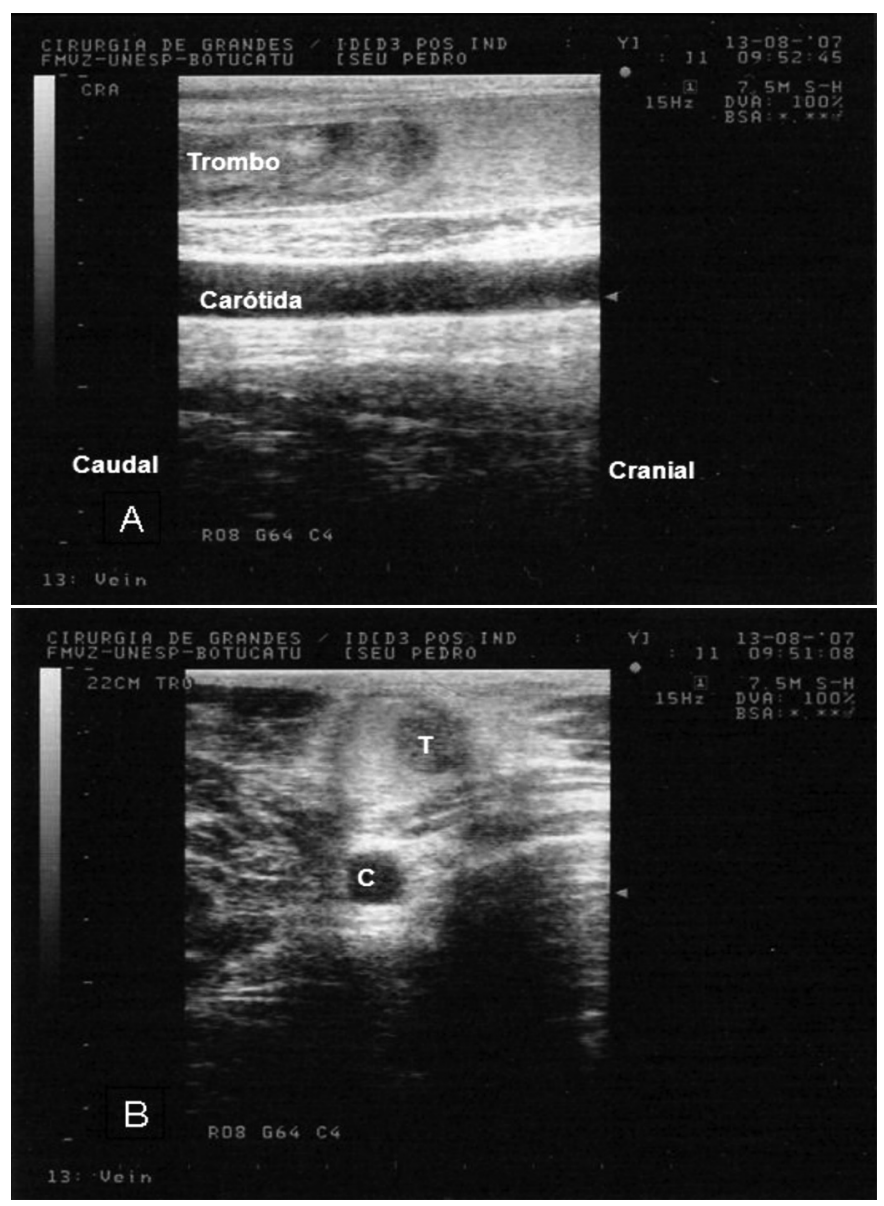

Fig.1. Aspecto ultra-sonográfico da veia jugular e estruturas relacionadas no período com tromboflebite, terceiro dia. (A) Corte longitudinal, à esquerda da cauda do trombo obstruindo a veia jugular, à direita sangue mais ecogênico. (B) Corte transversal mostrando o final da cauda do trombo (hipoecóica) circundada pelo sangue estagnado (mais ecóico). (C) Artéria carótida. Trombo dentro da veia jugular (T). 
As partes do trombo, cabeça, corpo e cauda, eram observáveis, com a cauda, por vezes livres e de fácil identificação. Em todos os animais os trombos se tornaram hiperecóicos e heterogêneos com a presença de áreas anecóicas, observável de modo evolutivo a cada avaliação diária, de modo evidente progredindo da região da cabeça para o corpo do trombo. Observou-se redução progressiva do diâmetro do trombo e da veia na região de indução.

As veias occipital, maxilar, linguofacial e o vaso colateral sem denominação anatômica mantiveram-se distendidos durante todo o período de indução em quatro animais.

0 vaso colateral sem denominação surgiu após a indução da tromboflebite e foi identificado em todos os animais durante todo o período de avaliação (Fig.2), visto na face dorsal da veia jugular próximo da abertura da veia linguofacial, visualizado com uma discreta inclinação dorsal do transdutor sobre a jugular no corte longitudinal. Este se unia a uma veia tributária longitudinal oriunda da região cefálica, paralelo à artéria carótida e seguia em sentido ao tórax.

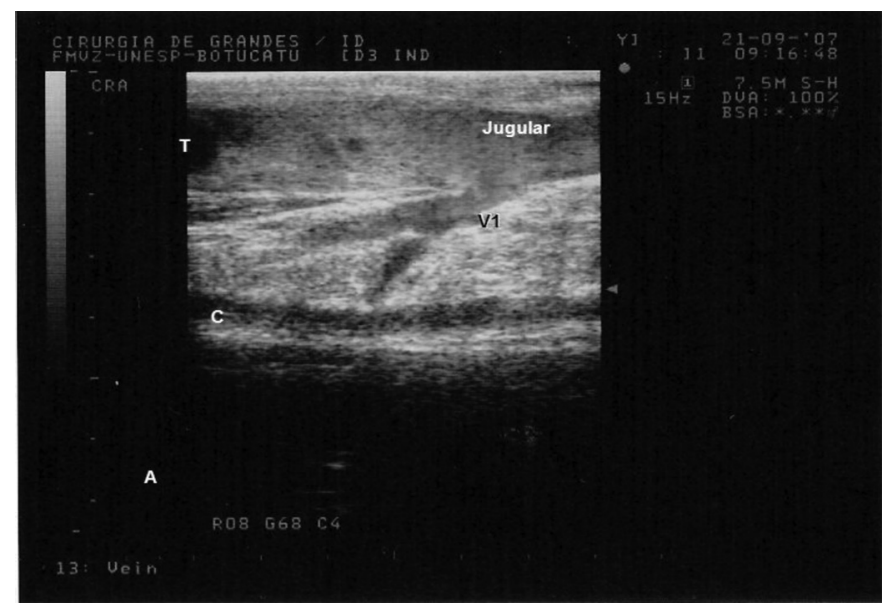

Fig.2. Imagem ultra-sonográfica no momento inicial de indução da tromboflebite, da porção cranial da veia jugular, observando-se o vaso colateral (V1) ramificando-se ventralmente. Trombo (T). Artéria carótida (C). Vaso colateral (V1).

Proximal à área de indução da tromboflebite observou-se diversas veias que chegavam à jugular e se ingurgitaram com a realização do garrote. 0 vaso longitudinal antes observado chegava nesta região e se distendia ainda mais com a realização do garroteamento jugular na entrada do torax. Em todos os animais houve a distensão da porção caudal à estenose com a realização de garrote na entrada do tórax, confirmando-se pelo exame ultra-sonográfico a presença e preenchimento venoso nesta porção jugular e confirmação de fluxo sanguíneo crânio-caudal.

A venografia antes dos procedimentos revelou imagens do fluxo laminar normal do sangue na veia jugular. Induzida a tromboflebite, dada a oclusão venosa o contraste se acumulou na porção cranial. Em um animal o contraste evidenciou as veias linguofacial e maxilar. Em outros dois animais o contraste seguiu até o terço médio da jugular. Em um animal o contraste circundou parte do trombo e pouco contraste foi visto entre a parede e o trombo. No sexto dia após induzida a tromboflebite observou-se de modo evi- dente a presença de vasos compensatórios que uniam as porções craniais às porções caudais ao trombo, na busca da drenagem sanguínea craniana pela veia jugular, com trajetos e comprimentos diversos e irregulares, sem evidencias de passagem de fluxo pela região com trombose. No $12^{\circ}$ dia observou-se a penetração do contraste no trombo. Houve animal em que o contraste contornou o lúmen e seguiu à porção caudal ao trombo. A presença de vasos se intensificou em diferentes formas e posições. No $18^{\circ}$ dia intensa malha vascular foi observada unindo as partes, cranial e caudal, ao segmento jugular com trombo. Observou-se ainda a passagem de contraste pelo trombo que permitiu a drenagem cefálica, associado aos vasos tributários descritos.

\section{DISCUSSÃO}

A tromboflebite jugular nos equinos está associada a processos iatrogênicos resultantes da utilização do vaso como principal acesso venoso no tratamento de enfermidades. $\mathrm{Na}$ ausência das veias jugulares profundas ou internas nesta espécie agravam-se os casos de trombose que podem levar ao óbito (Dornbusch et al. 2000, Hussni et al. 2009). A gravidade da enfermidade somada à necessidade de conhecer detalhes da evolução da tromboflebite nos equinos justifica este estudo que elucida parte das diversas questões sobre o tema e serve de base para a evolução nas pesquisas.

Os parâmetros clínicos temperatura corpórea, frequencias cardíaca e respiratória, coloração de mucosas e tempo de perfusão capilar mantiveram-se normais durante o experimento, em concordância com os animais utilizados, hígidos na indução do processo e controlado de modo asséptico e unilateral, seguindo a metodologia, e com resultados semelhantes ao descrito nas mesmas condições (Hussni et al. 2009).

Nos exames físicos da região cefálica, após a indução da tromboflebite o aumento de volume nas regiões massetérica, supraorbitária, submandibular e parotídea surgiram de modo evidente e reduziram com o tempo devendo-se considerar que os animais não foram tratados e que a tromboflebite foi unilateral, sugerindo que em condições associadas à toxemia ou outros fatores desencadeantes da tríade de Virschow o quadro clínico regional seria ainda mais intenso e persistente, diferente dos quadros complexos e bilaterais onde o agravamento se faz mais evidente e assim a redução das áreas com edema seria de menor resolução, que então levariam à evolução do quadro a partir do edema regional, asfixia, edema cerebral, culminando com o óbito (Rijkenhuizen \& Van Swieten 1998, Morris 1989, Hay 1998, Dornbusch et al. 2000, Hussni et al. 2009). No entanto, o sistema venoso dos equinos como em outras espécies possui capacidade compensatória de drenagem na obstrução de seus vasos maiores, com abundantes comunicações. A severidade e a persistência das alterações estão relacionadas ao numero e tamanho de vasos colaterais e anastomoses existentes (Gius \& Grier 1950). O fluxo sanguíneo poderá ser restaurado pela circulação colateral. Estas seriam veias não envolvidas na trombose que se distendem com o aumento de fluxo e passam a atuar como colaterais (Maffei et al. 2002) ou ainda o fluxo seria restaurado pela recana- 
lização do trombo. Salienta-se que as avaliações venográficas revelaram número crescente de vasos tributários na drenagem cefálica do lado da veia jugular com trombose, o que seguramente auxiliou na redução das áreas com edema e permitiu a passagem de sangue entre as porções cranial e caudal da veia com o ingurgitamento observado clinicamente e confirmado pelo exame ultra-sonográfico.

Na tromboflebite, o aumento de volume local com ingurgitamento do vaso e elevação da temperatura local (Morris 1989, Gardner et al. 1991, Dolente et al. 2005) mantiveram-se nos animais, que teve a isto somado à indução cirúrgica da tromboflebite intensificando o processo inflamatório local, mostrando sinais como sensibilidade dolorosa e aumento de temperatura local que regrediram rapidamente. 0 crescimento do trombo no sentido cranial observado clinicamente e confirmado por ultra-sonografia foi evidente, sem estar relacionado com seu desenvolvimento ou retra- ção quanto ao diâmetro, aspecto este não comparado.

No acompanhamento ultra-sonográfico da tromboflebite, estudos de casos de ocorrência natural em humanos sugerem que a recanalização começa rapidamente após um episódio de trombose venosa profunda, sendo encontrada em 7 dias em $40 \%$ dos pacientes, com a maior regressão do trombo ocorrendo em 3 meses, com recanalização completa em metade dos casos 6-9 meses após o evento trombótico (Meissner et al. 2007), mostrando esta evolução com aspectos similares mas diferindo por se tratar de outra espécie, etiologia e vaso comprometido.

A ecogenicidade do trombo foi semelhante ao descrito em ratos e primatas quanto à maturação deste (Fowlkes et al 1998), com o aumento da ecogenicidade à medida em que se torna maduro e poderia ser estabelecida a idade do trombo. Este aspecto não foi estudado por se tratar de experimento controlado quanto ao tempo de observação. A ocorrência de

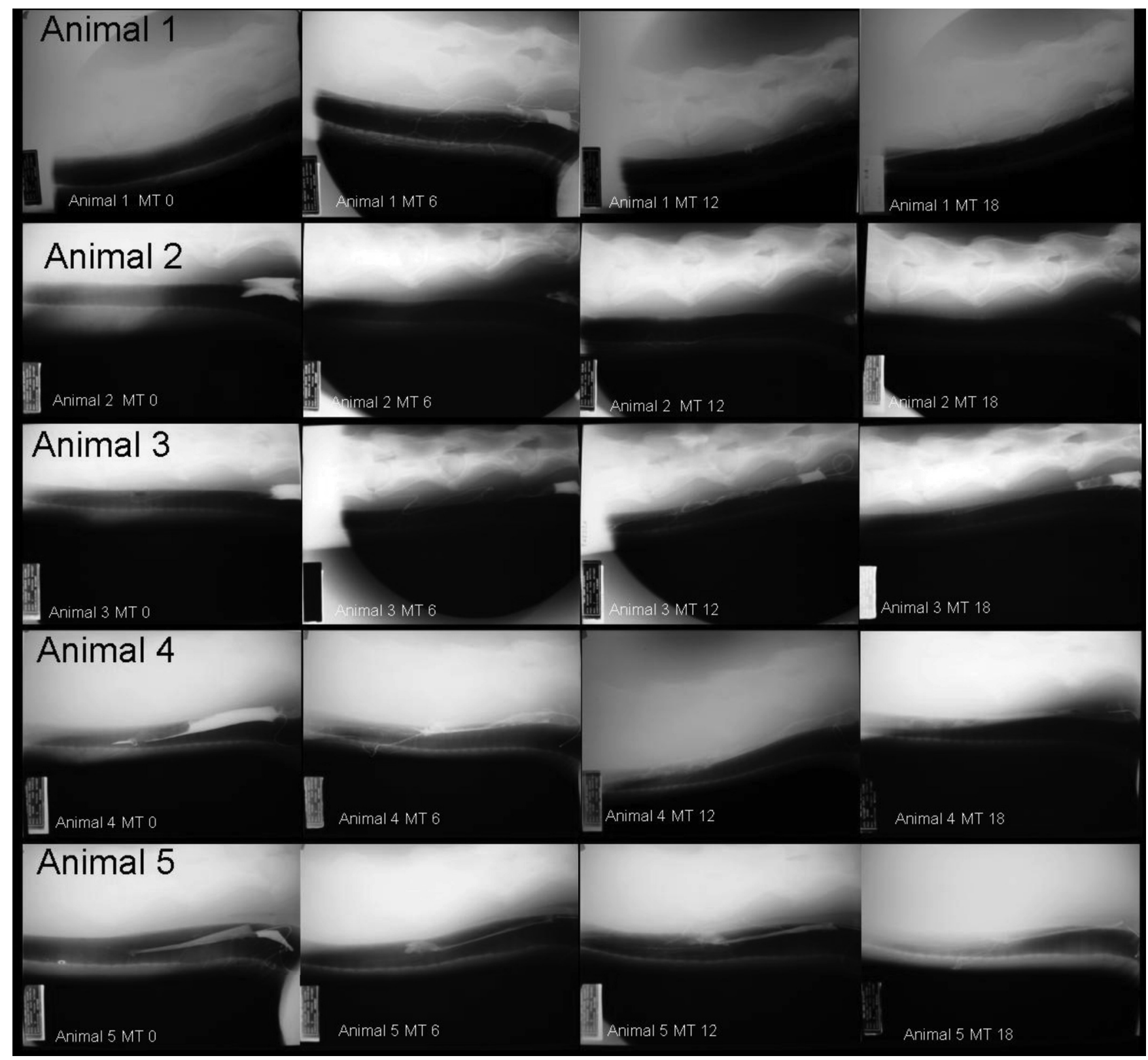

Fig.3. Venografias do período de tromboflebite nos dias 0 (inicial), 6, 12 e 18. 


\section{Quadro 1. Número de vasos tributários comunicantes das porções cranial a caudal ao trombo da veia jugular com tromboflebite, observados nas venografias nos animais nos diferentes momentos com a média e desvio padrão para cada momento nas fases de indução (FI) e após a indução da tromboflebite (TI)}

\begin{tabular}{lccccc}
\hline & FI imediato* $^{*}$ & FI final $(72 \mathrm{~h})$ & TI dia 6 & TI dia 12 & TI dia 18 \\
\hline Animal 1 & 0 & 5 & 10 & 9 & 8 \\
Animal 2 & 0 & 0 & 2 & 5 & 8 \\
Animal 3 & 1 & 7 & 7 & 5 & 5 \\
Animal 4 & 1 & 1 & 6 & 4 & 0 \\
Animal 5 & 0 & 17 & 8 & 13 & 8 \\
Média & 0 & 6 & 7 & 7 & 6 \\
DP & 1 & 7 & 3 & 4 & 3 \\
\hline
\end{tabular}

* Momento imediato à obstrução da veia.

regiões cavitárias hipoecogênica ou anecóicas foi associada à tromboflebite jugular séptica (Jesty \& Reef 2006), o que difere do observado que houve áreas anecóicas na maturação do trombo, sem sinais de infecção. Nos casos de trombose séptica haveria associação e agravamento dos achados clínicos e ultra-sonográficos. Enquanto o trombo progrediu em tamanho sofreu lise que é parte da reorganização observada, onde na recanalização permitiu a passagem de sangue, isto considerado como evolução na organização da trombose (Markel et. al. 2003, Meissner et al. 2007).

O exame venográfico forneceu informações sobre fluxo sanguíneo linear na higidez e na tromboflebite e expos a posição, extensão e formato da porção cranial do trombo. Ainda mostrou se este ocluía total ou parcialmente a luz venosa, bem como evidenciou os vasos colaterais tributários, sendo a venografia considerada por outros como útil, mas de pouca sensibilidade na detecção residual de trombos e no delineamento de canais microvasculares (Lin et al. 2001, Markel et al. 2003, Varma et al. 2003).

Os vasos colaterais tributários mostraram desenvolvimento em número e distribuição, sem, entretanto, ser possível afirmar se foram aberturas de vasos preexistentes, se eram neoformados ou ambas as condições. A não visualização de vasos tributários nos momentos finais de observação em animais com restabelecimento da perviedade, mesmo parcial da veia jugular com trombose, indicou que houve redução da pressão sanguínea de retorno e fechamento dos vasos tributários anteriormente ativos e observáveis à venografias.

\section{CONCLUSÕES}

Na tromboflebite jugular experimental induzida, a oclusão venosa pelo trombo sofreu recanalização, com a drenagem da região cefálica por vasos compensatórios à jugular ocluída.

Os métodos clínicos, ultra-sonográficos e venográficos permitiram a realização das avaliações e completaram-se na compreensão do processo.

A tromboflebite jugular induzida seguiu a evolução es- perada como enfermidade e serve de modelo para outros experimentos.

Agradecimentos.- À Coordenação de Aperfeiçoamento de Pessoal de Nível Superior (CAPES) pela bolsa e à Fundação para o Desenvolvimento da Unesp (Fundunesp) pelo auxílio concedido.

\section{REFERÊNCIAS}

Dolente B.A., Beech J., Lindborg S. \& Smith G. 2005. Evaluation of risk factors for development of catheter-associated jugular thrombophlebitis in horses: 50 cases (1993-1998). J. Am. Vet. Med. Assoc. 227:1134-1141.

Dornbusch P.T., Hussni C.A., Thomassian A., Alves A.L.G. \& Nicoletti J.L.M. 2000. Tromboflebite jugular nos equinos. Revta Educ. Contin. CRMV-SP 3:47-53.

Fowlkes J.B., Strieter R.M., Downing L.J., Brown S.L., Saluja A., Salles-Cunha S., Kadell A.M., Wrobleski S.K. \& Wakefield T.W. 1998. Ultrasound echogenicity in experimental venous thrombosis. Ultrasound Med. Biol. 24:1175-1182.

Gardner S.Y., Reef V.B. \& Spencer P.A. 1991. Ultrasonographic evaluation of horses with thrombophebitis of the jugular vein: 46 cases (1985-1988). J. Am. Vet. Med. Assoc. 199:370-373.

Gius J.A. \& Grier D.H. 1950. Venous adaptation following bilateral radical neck dissection with excision of the jugular veins. Surgery 28:305-321.

Hay W.P. 1998. Vascular reconstruction: Are we ready for the challenge? Equine Vet. J. 30:178-179.

Hussni C.A., Dornbusch P.T., Yoshida W.B., Alves A.L.G., Nicoletti J.L.M., Mamprim M.J. \& Vulcano L.C. 2009. Trombectomia com cateter de Fogarty no tratamento da tromboflebite jugular experimental em eqüinos. Pesq. Vet. Bras. 29:45-51.

Jesty S.A. \& Reef V.B. 2006. Septicemia and Cardiovascular Infections in Horses. Vet. Clin. North Am., Equine Pract. 22:481-495.

Lin P.H., Chen C., Surowiec S.M., Conklin B., Bush R.L. \& Lumsden A.B. 2001. Evaluation of thrombolysis in a porcine model of chronic deep venous thrombosis: An endovascular model. J. Vasc. Surg. 33:621-627.

Maffei F.H.A., Lastoria S., Yoshida W.B. \& Rollo H.A. 2002. Doenças Vascula-

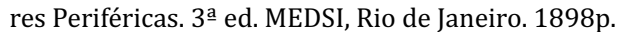

Markel A., Meissner M., Manzo R.A., Bergelin R.O. \& Strandness Jr D.E. 2003. Deep venous thrombosis: rate of spontaneous lysis and thrombus extension. Int. Angiol. 22:376-82.

Meissner M.H., Wakefield T.W., Ascher E., Caprini J.A., Comerota A.J., Eklof B., Gillespie D.L., Greenfield L.J., Ruth H.E., Henke P.K., Hingorani A., Hull R.D., Kessler C.M., McBane R.D. \& McLafferty R. 2007. Acute venous disease: Venous thrombosis and venous trauma. J. Vasc. Surg. 46:25-53.

Montenegro M.R. \& Franco M. 1999. Patologia: processos gerais. $4^{\mathrm{a}}$ ed. Atheneu, São Paulo. 292p.

Morris D.D. 1989. Thrombophlebitis in horses: The contribution of hemostatic disfuntion to pathogenesis. Contin. Educ. 11:1386-1395.

Rijkenhuizen A.B.N. \& Van Swieten H.A. 1998. Reconstruction of the jugular vein in horses with post thrombophlebitis stenosis using saphenous vein graft. Equine Vet. J. 30:236-239.

Ryu S., Kim J., Bak U., Lee C. \& Lee Y.L. 2004. A hematogenic pleuropneumonia caused by postoperative septic thrombophlebitis in a Thoroughbred gelding. J. Vet. Sci. 5:75-77.

Varma M.R., Varga A.J., Knipp B.S., Sukheepod P., Upchurch G.R., Kunkel S.L., Wakefield T.W. \& Henke P.K. 2003. Neutropenia impairs venous thrombosis resolution in the rat. J. Vasc. Surg. 38:1090-1098.

Wiemer P., Gruys E. \& Van Hoeck B. 2005. A study of seven different types of grafts for jugular vein transplantation in the horse. Res. Vet. Sci. 79:211-217. 